\title{
Strand Movements in Cable-in-Conduit Conductors
}

\author{
Kazuya Takahata and Takashi Satow
}

\begin{abstract}
The compression tests with dummy bundles have been performed to investigate the strand movements in cable-in-conduit conductors. The bundle consists of $20 \mathrm{~mm}$ long vinyl tubes. The cross section of the bundle is a round-cornered rectangle of $10 \mathrm{~mm} \times 20 \mathrm{~mm}$. Body force was applied in the transverse direction by means of a pressurized argon gas flow at room temperature. Pressure gradient in the bundle produced body force acting on each strand. The strand movements were observed with a CCD camera. Surface pressure was also applied with a piston, and a comparison has been made between two methods. Influence of a sub-bundle structure on the movements is also investigated.
\end{abstract}

Index Terms-Body force, cable-in-conduit conductor, compression test, strand movements.

\section{INTRODUCTION}

A CABLE-IN-CONDUIT conductor is the most promising candidate for use in a fusion magnet because of high stiffness and dielectric strength. However, some of the problems remain to be solved concerning $\mathrm{AC}$ losses, imbalanced current distribution and strand movements due to electromagnetic force. In particular, quantitative treatments on the strand movements are not yet well developed. The strand movements can cause point disturbances, increase of AC losses, and change of hydraulic properties. In the previous works, transient disturbances have been investigated with a compression test of a multi-strand cable and an acoustic emission technique by Schauer [1]. Effect on transverse conductance among strands has been reported by Ono et al. [2]. In the experimental investigations, the cables were compressed by surface pressure instead of body force. Recent work by Nishijima et al. has developed the Monte Carlo method to calculate strand movements [3]. In the analyses, a compressive load is applied as body force. As compared with the load-deformation curves presented in the papers, the curves show some different features. Differences in loading methods are considered to affect strand movements. To perform a compression test with electromagnetic force, a large device including a magnet, power supply, and refrigerant must be necessary. In addition, it is difficult to observe the movements of each strand.

In this work, the strand movements in multi-strand bundles have been investigated experimentally. Compressive load was applied to the bundle with not only surface pressure but also body force by using a newly developed method. Differences in the strand movements between two compressive methods are in-

Manuscript received September 24, 2001. This work was supported in part by the Japan Society for the Promotion of Science, Grant-in-Aid for Scientific Research (c), 13680573, 2001.

The authors are with the National Institute of Fusion Science, Toki, Gifu 509-5292 Japan (e-mail: takahata@LHD.nifs.ac.jp; satotaka@LHD.nifs.ac.jp).

Publisher Item Identifier S 1051-8223(02)04279-3.

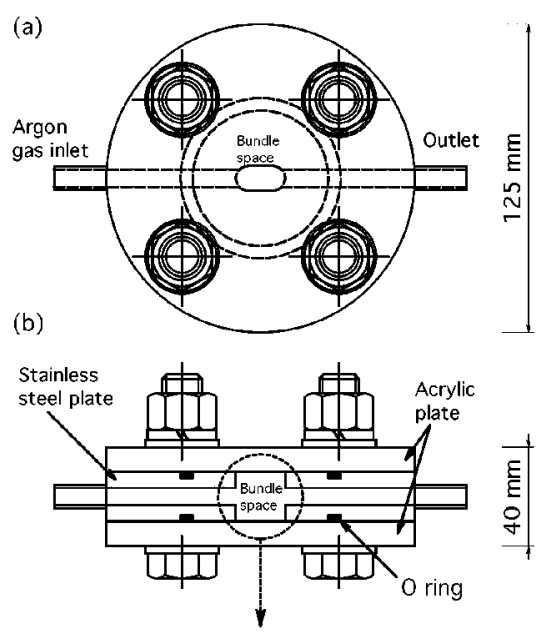

(c)

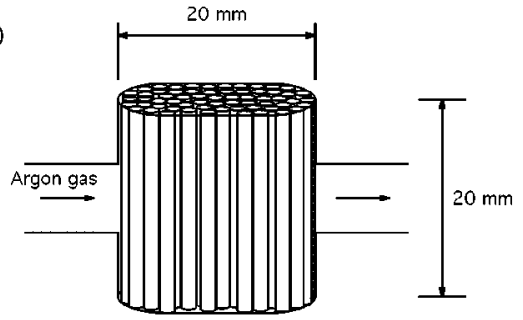

Fig. 1. Setup of the compression test. (a) Top and (b) side views of the sample holder, and (c) close-up of the bundle space.

vestigated. The effect of the sub-bundle structure on the movements is also presented.

\section{EXPERIMENTAL PROCEDURES}

\section{A. Bundle and Sample Holder}

The bundle consists of vinyl tubes $20 \mathrm{~mm}$ in length, 1.52 and $1.08 \mathrm{~mm}$ in diameter. The cross section of the bundle is a round-cornered rectangle of $10 \mathrm{~mm} \times 20 \mathrm{~mm}$. The bundle is put in a sample holder as shown in Fig. 1. The holder has the bundle space and gas inlet and outlet pipes and holes $7 \mathrm{~mm}$ in diameter. The section of the bundle and the strand movements can be observed through acrylic flanges with a CCD camera.

Four types of bundles were prepared. The bundles consist of different components as shown in Table I. The strands were bonded with an adhesive to make the sub-bundle. The strand fraction, $f_{s}$ is defined as the ratio of the area occupied by strands without the adhesive to the bundle space area.

\section{B. Compressive Load}

In the first experiment, the surface pressure was applied by using a piston as shown in Fig. 2(a). The area on which pres- 
TABLE I

COMPONENTS OF BUNDLE

\begin{tabular}{llcc}
\hline Sample Code & Component of Bundle & Strand Diameter \\
\hline 1S & Single strand & 0 & $1.52 \mathrm{~mm}$ \\
3S & Sub-bundle of 3 strands & $\varnothing$ & $1.52 \mathrm{~mm}$ \\
4S1 & Sub-bundle of 4 strands & $\varnothing 8$ & $1.52 \mathrm{~mm}$ \\
4S2 & Sub-bundle of 4 strands & 88 & $1.08 \mathrm{~mm}$ \\
\hline
\end{tabular}

(a)

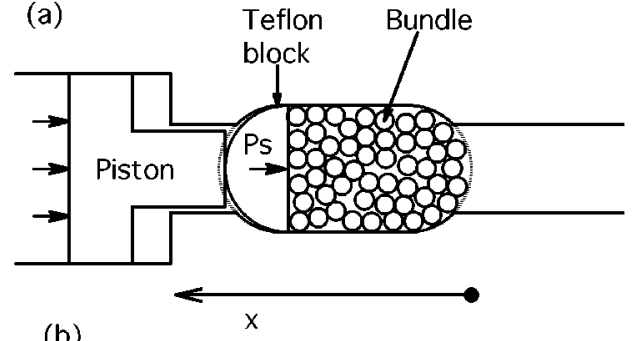

(b)

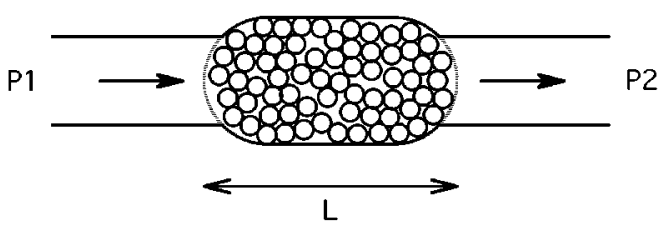

Fig. 2. Test procedure of the compression tests with (a) surface pressure and (b) body force.

sure, $P s$ acts is $10 \mathrm{~mm} \times 20 \mathrm{~mm}$. In the second experiment, the body force was applied by a pressurized argon gas flow in the transverse direction as shown in Fig. 2(b). The inlet pressure, $P 1$ can be increased up to $0.6 \mathrm{MPa}$. The outlet pressure, $P 2$ is atmospheric. Fig. 3 shows the relation between the pressure drop, $(P 1-P 1)$ and the mass flow rate. The curve A shows the result for the sample $3 S\left(f_{s}=0.73\right)$. When the flow through gaps between the bundle and the acrylic plates was stopped by clay, the curve $B$ was obtained. A comparison between the curve $A$ and $B$ shows that most of the gas flows through the gaps as shown in Fig. 4. The gap width is estimated to be approximately $0.1 \mathrm{~mm}$. The pressure drop along the gaps may generate the pressure distribution in the bundle. The pressure gradient then produces the body force acting on each strand. The force acting on a strand, $F$ is similar to buoyancy, and simply obtained by

$$
F=\pi r^{2}(P 1-P 2) / L
$$

where $r$ is the radius of strand, and $L$ is the length of bundle. Here the linear pressure gradient is assumed. With $r=0.76 \mathrm{~mm}, P 1-P 2=0.5 \mathrm{MPa}$, and $L=20 \mathrm{~mm}$, (1) shows $F$ to be $45 \mathrm{~N} / \mathrm{m}$. On the other hand, the maximum electromagnetic force applied to a strand is about $300 \mathrm{~N} / \mathrm{m}$ in the case of poloidal field coils for Large Helical Device [4]. It is difficult to apply the force equivalent to the electromagnetic field. Therefore, the rigidity of the bundle is reduced by using

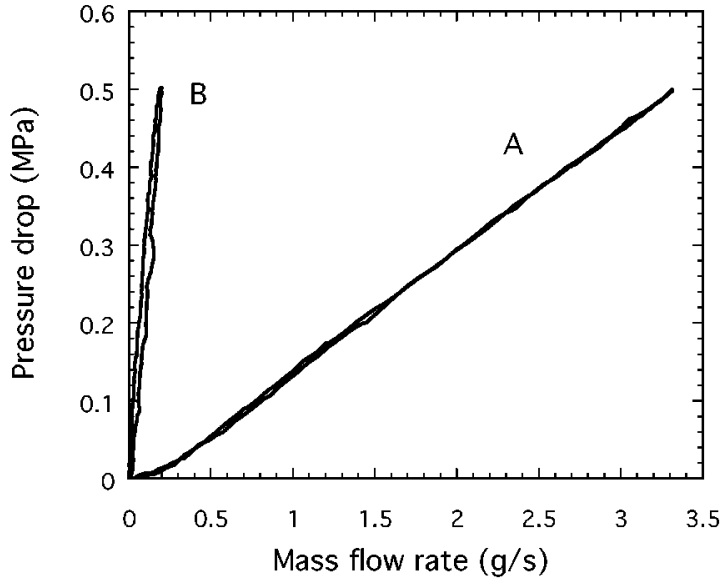

Fig. 3. Pressure drop in the bundle space. The sample is $3 S\left(f_{s}=0.73\right)$. The pressure drop changes from $A$ to $B$ by stopping the flow through the narrow gaps near the acrylic plates.

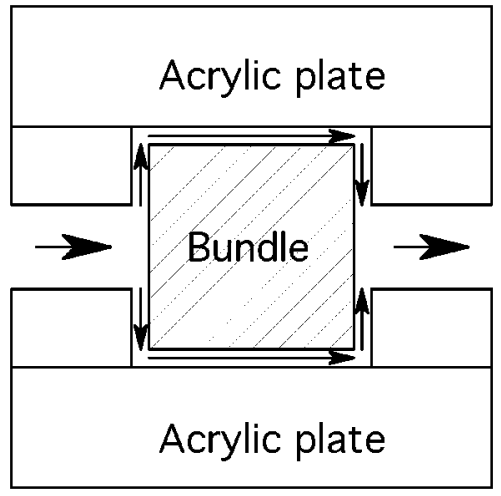

Fig. 4. Schematic diagram of the gas flow in the bundle space.

soft strands, and the equivalent strand displacements can be generated. The previous studies have estimated the cable deformation to be approximately $1 \mathrm{~mm}$ [2], [3].

\section{RESULTS AND DISCUSSION}

\section{A. Load-Displacement Curves}

In the first step, the compression tests were performed with the sample $3 S$. Because most cable-in-conduit conductors consist of triplet, the sample $3 \mathrm{~S}$ is considered to have similar mechanical properties to the actual cable. Fig. 5 shows the load-displacement curves for the bundle $\left(f_{s}=0.66\right)$ subjected to surface pressure. To define the average displacement, the absolute values of displacements, $\mathrm{s}$ for all strands are plotted as a function of the position, $x$ which is defined in Fig. 2. The average displacement is then obtained as the slope of the straight line approximated by the least squares method. Fig. 6 shows the load-displacement curves for the bundle $\left(f_{s}=0.64\right)$ subjected to body force. At the beginning of compression in the first cycle, low rigidity and nonelastic region was observed for both tests. In this region, contacts among adjacent strands may occur. After all strands 


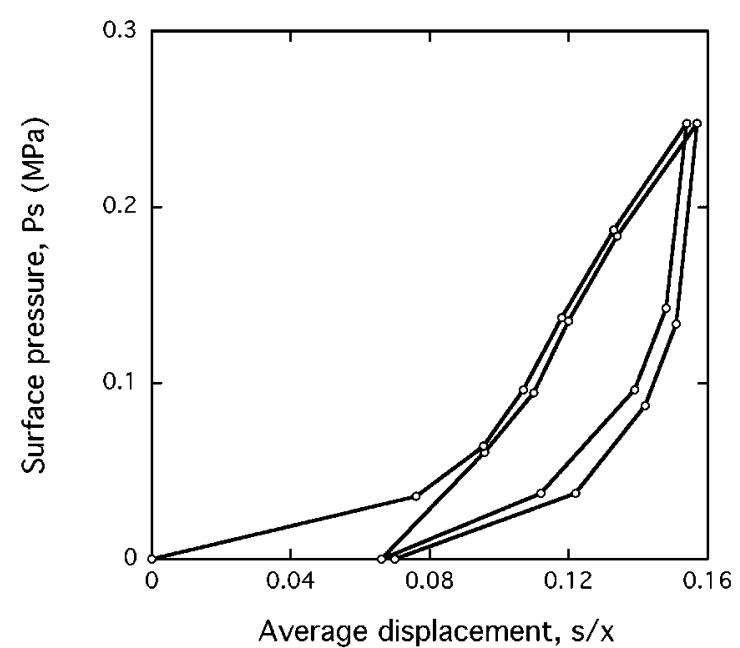

Fig. 5. Load-displacement curves for the compression test with surface pressure. The sample is $3 S\left(f_{s}=0.66\right)$.

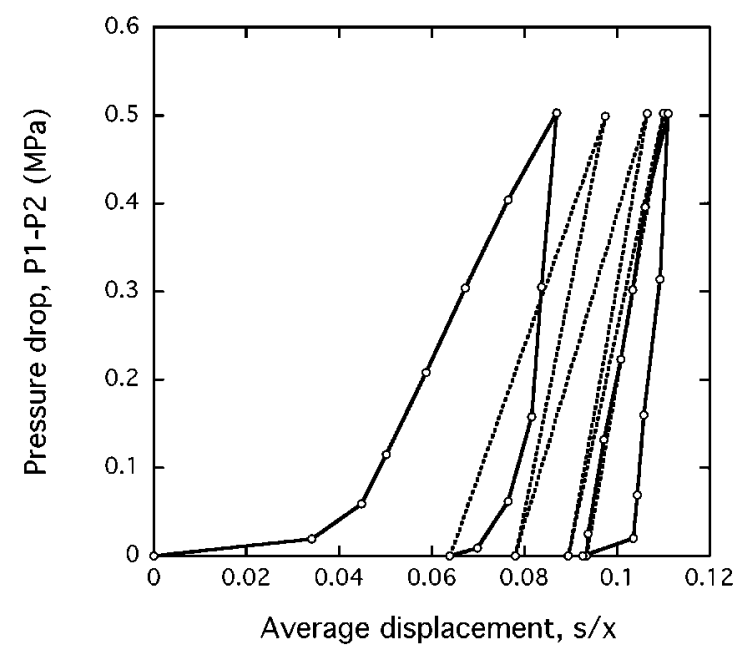

Fig. 6. Load-displacement curves for the compression test with body force. The sample is $3 S\left(f_{\mathcal{s}}=0.64\right)$.

were contacted, the rigidity increased. On the surface pressure test, pseudoelastic hysteresis loop was observed from the second cycle. On the body force test, the nonelasticity appeared until the fourth cycle. In addition, the rigidity increased with the number of cycles. The analytical results show a similar tendency [3]. It was found by detail observations that some strands moved not only in the direction opposite to $x$ but also in the perpendicular direction and also rotated while the pressure drop increased. In the case of body force, the transmission of force influences the displacements. The increase of contact points may cause the change of force transmission and the subsequent movements. In the case of surface pressure test, the strands moved only in the direction opposite to $x$. Factors determining the displacements may be only the deformation of strands and the friction among adjacent strands.

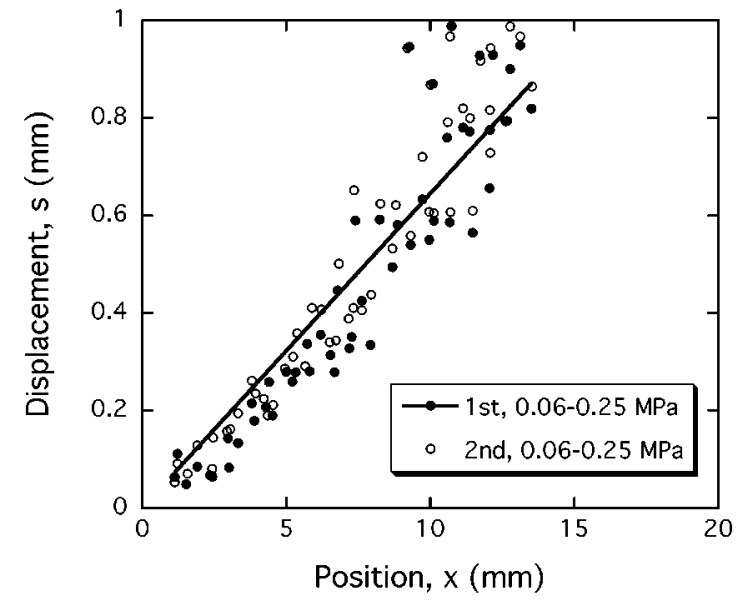

Fig. 7. Displacements of the strands for the compression test with surface pressure.

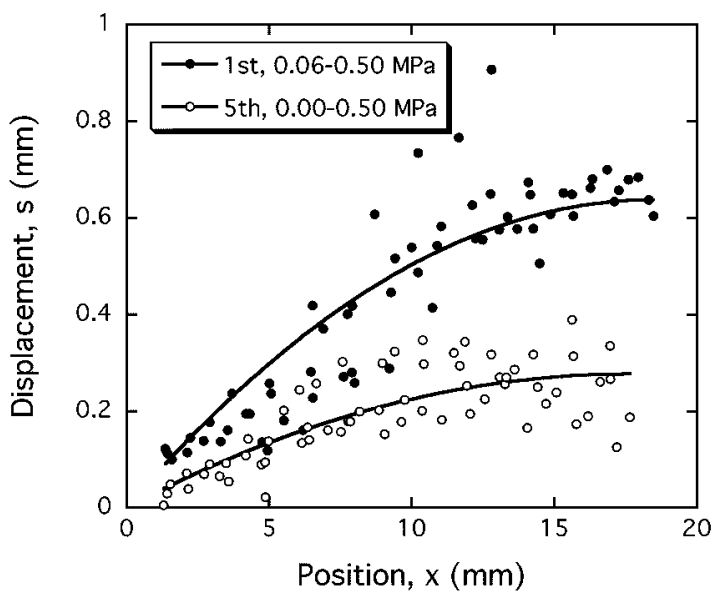

Fig. 8. Displacements of the strands for the compression test with body force.

\section{B. Displacements of Strands}

Assuming the bundle is a homogeneous substance, and the apparent modulus, $E$ is constant, the absolute value of the displacement, $s$ can be expressed as,

$$
\begin{aligned}
& s=(P s / E) x \\
& s=\left[f_{s}(P 1-P 2) /(2 E L)\right]\left[L^{2}-(L-x)^{2}\right]
\end{aligned}
$$

for the surface pressure and the body force, respectively. The displacements of all strands are plotted in Figs. 7 and 8 in the regions where the apparent modulus is comparatively constant. The number of cycle and the pressure range are shown in the figures. On the surface pressure test, the displacements are directly proportional to $x$. This agrees with (2). In Fig. 8, the regression curves of (3) are plotted. The displacements show almost the same dependence as (3). It is confirmed that the body force can be applied to the bundle by this method. It is also clear that the modulus increased with the number of cycles. The apparent 


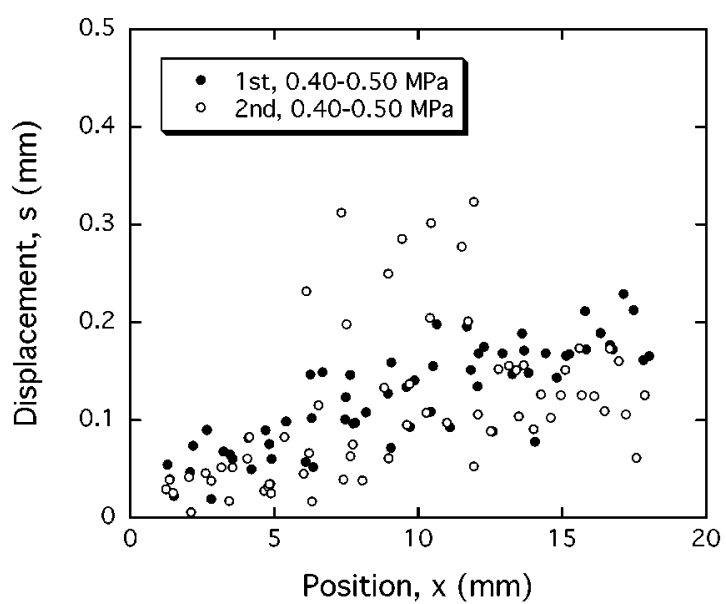

Fig. 9. Displacements of the strands in the large-force region.

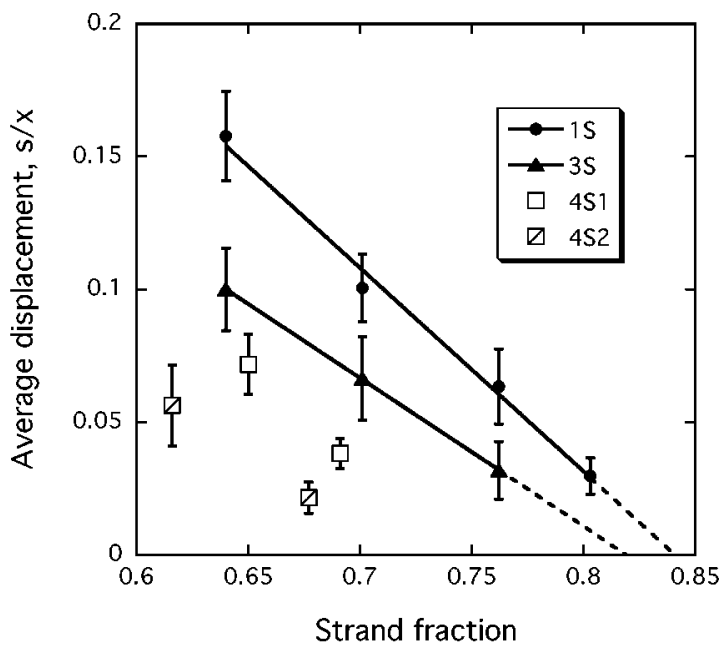

Fig. 10. Effect of the sub-bundle structure on the strand displacement. The sample codes are defined in Table I.

modulus can be obtained by (2) and (3). The moduli obtained from the regression curves are approximately 3.0 and $4.1 \mathrm{MPa}$ for the first cycle of compressions with the surface pressure and body force, respectively. It shows relatively good agreement.

During the body force tests, it was found that some strands occasionally showed quick movements in the large-force region of the second cycle instead of the first cycle. The displacements in the first and second loading are shown in Fig. 9. The range of pressure drop is from 0.40 to $0.50 \mathrm{MPa}$. Although the displacements of most strands are reduced, several strands around the center show large movements. This may be caused by the previously described change of force transmission. The results suggest that the local disturbance causing instability can occur even if the excitation is the second run.

\section{Effect of Sub-Bundle Structure}

To examine the effect of sub-bundle structure, four types of bundles were prepared as shown in Table I. The average displacements were measured when the pressure drop of $0.5 \mathrm{MPa}$ were applied to the samples. Fig. 10 shows the displacements as a function of strand fraction. More than five samples were tested under the same condition. The displacements of $1 S$ and $3 S$ decreased linearly with increasing the strand fraction. It should be noted that the displacements were reduced where the bundle consisted of the sub-bundle of four strands ( $4 S 1$ and $4 S 2$ ). In this case, it is difficult to form the closest packing of triangular lattice. On the other hand, the sample $3 S$ can easily produce the triangular lattice, which gives large packing density. That is why the sample $3 S$ shows large strand displacements. Considering actual conductors, the sub-cables of most cable-in-conduit conductors are triplet. However, the results imply that sub-cable of four strands is effective to reduce the strand movements.

\section{CONCLUSIONS}

The compression tests with dummy bundles have been performed to investigate the strand movements in cable-in-conduit conductors. Not only surface pressure but also body force could be applied to the sample by using a pressurized gas flow. During several compression cycles by the body force, irreversible displacements of the strands were observed, and the rigidity of the bundle increased with the number of cycles. This is caused by the increase of contact points, the change of transmission of force, and the subsequent movements. It is suggested that instability due to the strand movements might occur even at the second and later excitations. It was also found that the structure of sub-bundle affected the strand movements. To reduce the movements, the optimum structure of sub-cables may exist for cable-in-conduit conductor. In this work, an advantage of sub-cables of four strands was suggested.

\section{REFERENCES}

[1] F. Schauer, "Optimization and stability of a cable-in-conduit superconductor," IEEE Trans. Magn., vol. 32, pp. 2760-2763, Jul. 1996.

[2] M. Ono et al., "The relation between transverse mechanical and electrical properties of the multi-twisted stage cables of CICC," IEEE Trans. Appl. Superconduct., vol. 7, pp. 808-811, Jun. 1997.

[3] S. Nishijima, T. Sasaki, K. Takahata, and T. Satow, "Monte Carlo calculation of strand position in CIC conductor to analyze mechanical loss," IEEE Trans. Appl. Superconduct., vol. 11, pp. 1462-1465, Mar. 2001.

[4] M. Fujiwara et al., "Large helical device (LHD) program," J. Fusion Energy, vol. 15, pp. 7-154, 1996. 